\title{
RANS Simulations of Flow Past an DU-91-W2-250 Airfoil at High Reynolds Number
}

\author{
Krzysztof Rogowski ${ }^{1, *}$, and Martin O.L. Hansen ${ }^{2}$ \\ ${ }^{1}$ Institute of Theoretical and Applied Mechanics, Warsaw University of Technology, Warsaw, \\ 00-665, Poland \\ ${ }^{2}$ DTU Wind Energy, Technical University of Denmark, Lyngby, 2800 Kgs., Denmark
}

\begin{abstract}
This paper presents numerical results of the DU-91-W2-250 airfoil. Reynolds-averaged Navier-Stokes (RANS) simulations of the 2D profile are performed employing the Transient SST turbulence model. The airfoil was investigated for the Reynolds number of $6 \cdot 10^{6}$. Lift and drag coefficients are compared with the experimental data from LM Low Speed Wind Tunnel (LSWT). The results of lift and drag coefficients obtained using the SST Transient model are in a good agreement in comparison with the experiment in the angle of attack range from $-10^{\circ}$ to $10^{\circ}$. The static pressure distributions calculated by the SST Transition model are also in good agreement with the experiment.
\end{abstract}

\section{Introduction}

A few decades ago it was common to use the NACA family airfoils for design of wind turbines. However, they were not very effective for these applications. The development of the computer industry led to the development of numerical tools for the design of aerodynamic airfoils. In 1984 the National Renewable Energy Laboratory (NREL) was one of the first institution to start research on special-purpose airfoils for horizontal-axis wind turbines (HAWTs). During this investigation a few airfoil families were designed for different rotor sizes. The purpose of these investigations was to achieve a maximum lift coefficient which is less sensitive to roughness effects. During operation, a wind turbine exhibits variable rpm, variable pith and stall and the airfoil design should take into account such factors. In the case of stall-regulated rotors, an appropriate design of tip airfoils should restrain the maximum lift coefficient, however it allows for better peak-power control. The advantage of the tip airfoils with a large value of the maximum lift coefficient is the possibility of the use of lightweight blades with low solidity in variable-rpm and variablepitch wind turbines. Blades having full-span pitch control exhibit less drag for tip airfoils with low thickness [1]. Airfoils of modern large wind turbines are designed in such way that the rotor power coefficient is large and chord length is small to ensure high turbine performance and at low costs [2]. Delft University of Technology (DUT) investigated a series of DU-airfoils with maximum thickness from $15 \%$ to $40 \%$ using a wind tunnel and the modified version of XFOIL. The influence of the trailing edge wedges, Gurney flaps and

* Corresponding author: krogowski@meil.pw.edu.pl 
vortex generators on the airfoil performance was also analysed experimentally. Airfoils designed by Delft University of Technology are used in small and large wind turbines ranging from $350 \mathrm{~kW}$ to $3.5 \mathrm{MW}$. A DU 91-W1-251 airfoil was designed in order to avoid shortcomings in the NACA series profiles. This deficiency was low airfoil performances near the blade root due to premature transition [3]. The influence of the vortex generators on the a DU-91-W2-250 airfoil was investigated in wind tunnel at LM Wind Power [4]. Also Risoe National Laboratory [5] described the wanted properties for airfoils to be applied on wind turbines and used these to design dedicated airfoil geometries.

Nowadays, computational methods of fluid dynamics have become a very important tool in the design of aerodynamic airfoils. Reynolds-averaged Navier-Stokes (RANS) simulations of the DU 91-W2-250 airfoil were performed by Bertagnolio et al. [6] employing the $k-\omega S S T$ turbulence model. The obtained results were compared with the experimental data for the Reynolds of $1.0 \cdot 10^{6}$. Usually, RANS modelling is appropriate for linear part of the lift coefficient curve. For angles of attack, higher than critical angles of attack the large eddy simulation (LES) approach is required. LES model allows to resolve a wide range of time and length scales, however, this approach is very expensive computationally [7], however this is still much less expensive than obtaining aerodynamic coefficients from flight data $[8]$.

In this paper a steady-state compressible Reynolds-averaged Navier-Stokes equations with the SST Transition turbulence model are used for analysis of flow past the DU-91-W2250 airfoil at the Reynolds number of $6 \cdot 10^{6}$.

\section{Numerical model}

Figure 1 presents the shape of the DU-91-W2-250 airfoil designed by Timmer. The relative thickness of the airfoil as percent of its chord is $25 \%$. The following design aims were taken into account during the design process: the maximum lift coefficient of approx. 1.5, insensivity to roughness and relatively smooth stall [5].

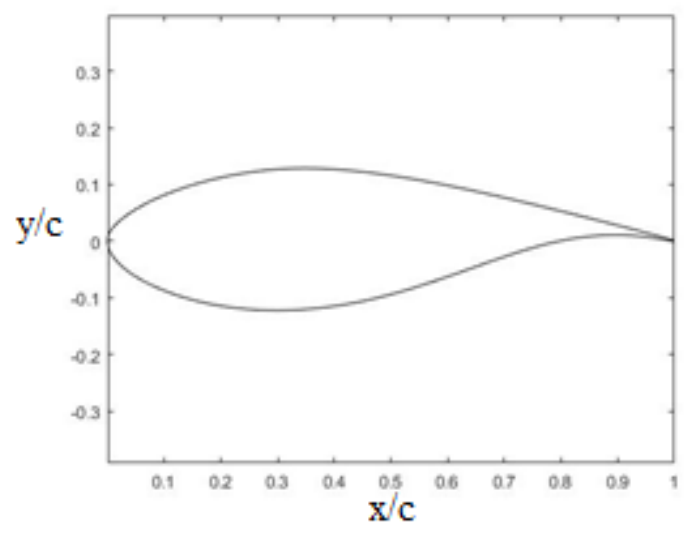

Fig. 1. An DU-91-W2-250 airfoil.

The measurements were performed at the LM Low Speed Wind Tunnel (LSWT). This closed loop type wind tunnel is equipped with a $1 \mathrm{MW}$ fan and a $2.7 \mathrm{~m} \times 1.35 \mathrm{~m} \times 7 \mathrm{~m}$ test section. The flow velocity range in the wind tunnel is up to $105 \mathrm{~m} / \mathrm{s}$ whereas the turbulence intensity is less than $0.05 \%$. The measurements were performed in May 2015 using the blade with DU-91-W2-250 profile. The chord length of the airfoil was $0.9 \mathrm{~m}$. The blade is made with carbon fiber. In order to measure lift coefficients, 62 pressure taps were placed in the 
blade. Lift forces are calculated by integrating the obtained static pressure distributions whereas the drag bucket is measured using a traversing wake rake.

In this paper, Reynolds-averaged Navier-Stokes (RANS) simulations of the DU-91-W2250 airfol are performed for Reynolds number of $6 \cdot 10^{6}$ and Mach number of 0.3 . For these flow conditions the free stream velocity is approx. $102 \mathrm{~m} / \mathrm{s}$. Turbulence intensity is assumed the same as in the experiment. CFD simulations are performed for the angle of attack in the range between $-12^{\circ}$ and $12^{\circ}$.

\section{Method}

The C-mesh is used for all simulations presented in this paper. The distances from the computational domain boundaries to the airfoil are $20 \mathrm{~m}$ ( 20 chord lengths). The generated mesh consists of 145,600 structured grid cells. The size of the first cell at the profile boundary is $3 \mathrm{e}-7 \mathrm{~m}$ providing wall $\mathrm{y}^{+}$values of approx. 1.3. At the Reynolds number of $6 \cdot 10^{6}$ the mesh is fine enough to resolve the viscos-dominated sublayer. The number of cells on the airfoil surface is 800 . Further refinements to the mesh did not give better results of aerodynamic blade loads and pressure distributions. Figure 2 presents mesh distribution for the DU-91W2-250 airfoil.

The computations were performed using the density-based solver with implicit formulation method. The gradients are computed employing the Green-Gauss Node-Based method. For the governing and turbulence equations the second order upwind schemes were used.

In this investigation the Transition shear stress transport (SST) turbulence model was employed to obtain turbulence quantities. This four-equation turbulence model is based on the SST k- $\omega$ model and two transport equations for the intermittency and for the transition onset criteria. For more information see ANSYS FLUENT Theory Guide 15.0.

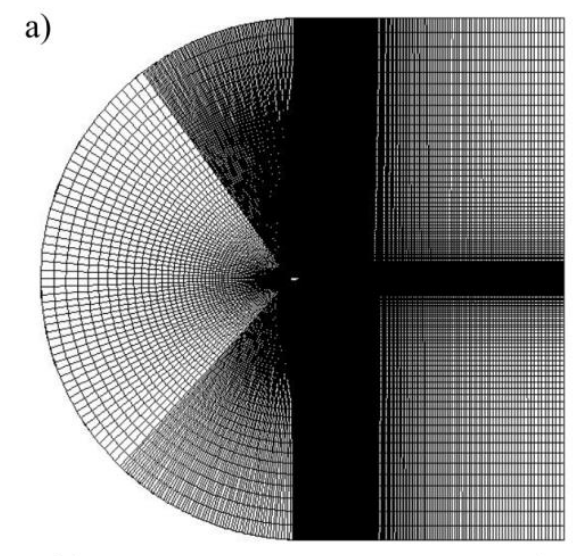

b)

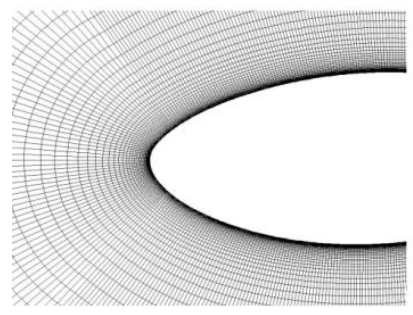

c)

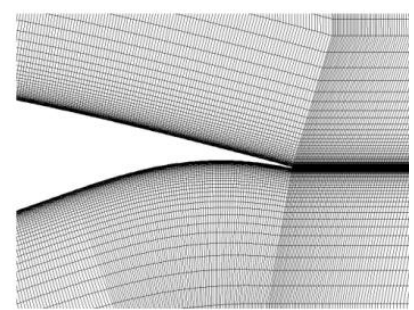

Fig. 2. Mesh. 


\section{Results and discussion}

Lift and drag force coefficients, $C_{L}$ and $C_{D}$, are dimensionless coefficients defined as:

$$
L, D=0.5 \cdot C_{L, D} \cdot A \cdot \rho \cdot V^{2}
$$

where: $L$ and $D$ are lift force and drag, respectively; $\rho$ is the air density; $V$ is the flow velocity at the inlet and $A$ is the blade reference area. The reference area is determined as: $A=c \cdot 1$, where $\mathrm{c}$ is the length chord. Lift force and drag depend on the Reynolds number, Re, and the angle of attack, $\alpha$. Figures 3 and 4 show respectively lift and drag coefficients as functions of the angle of attack. The characteristics are computed for the angle of attack range between $-12^{\circ}$ and $12^{\circ}$. As it can be seen from Fig. 3, the obtained results of lift coefficients are in a good agreement with the experimental data in the linear part of the characteristic curve. The experimental values of the maximum lift coefficients, for the positive and negative critical angles of attack, are not predicted by RANS simulations. The drag values are slightly overestimated compared with the experiment for positive values of the angle attack. For high angles of attack, the drag coefficients, computed by CFD, are underestimated.

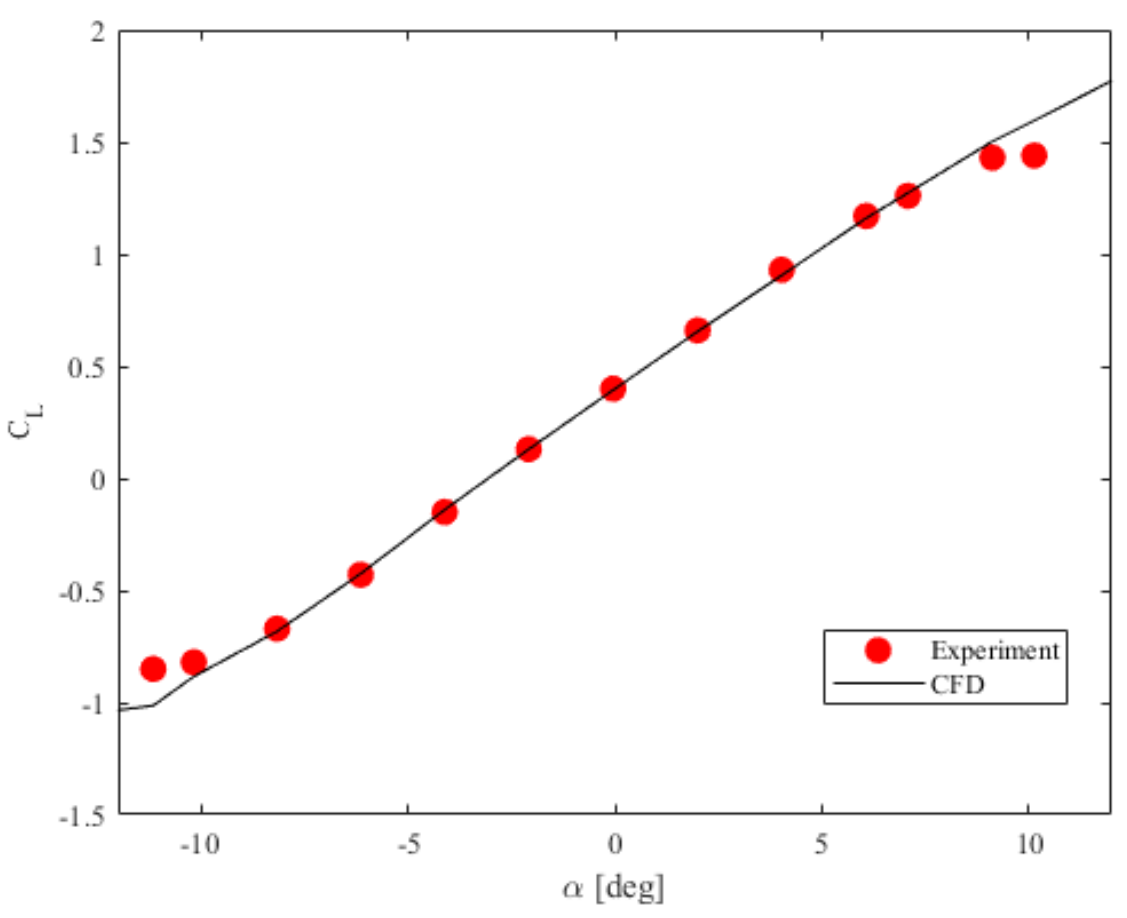

Fig. 3. Lift coefficient vs. angle of attack. 


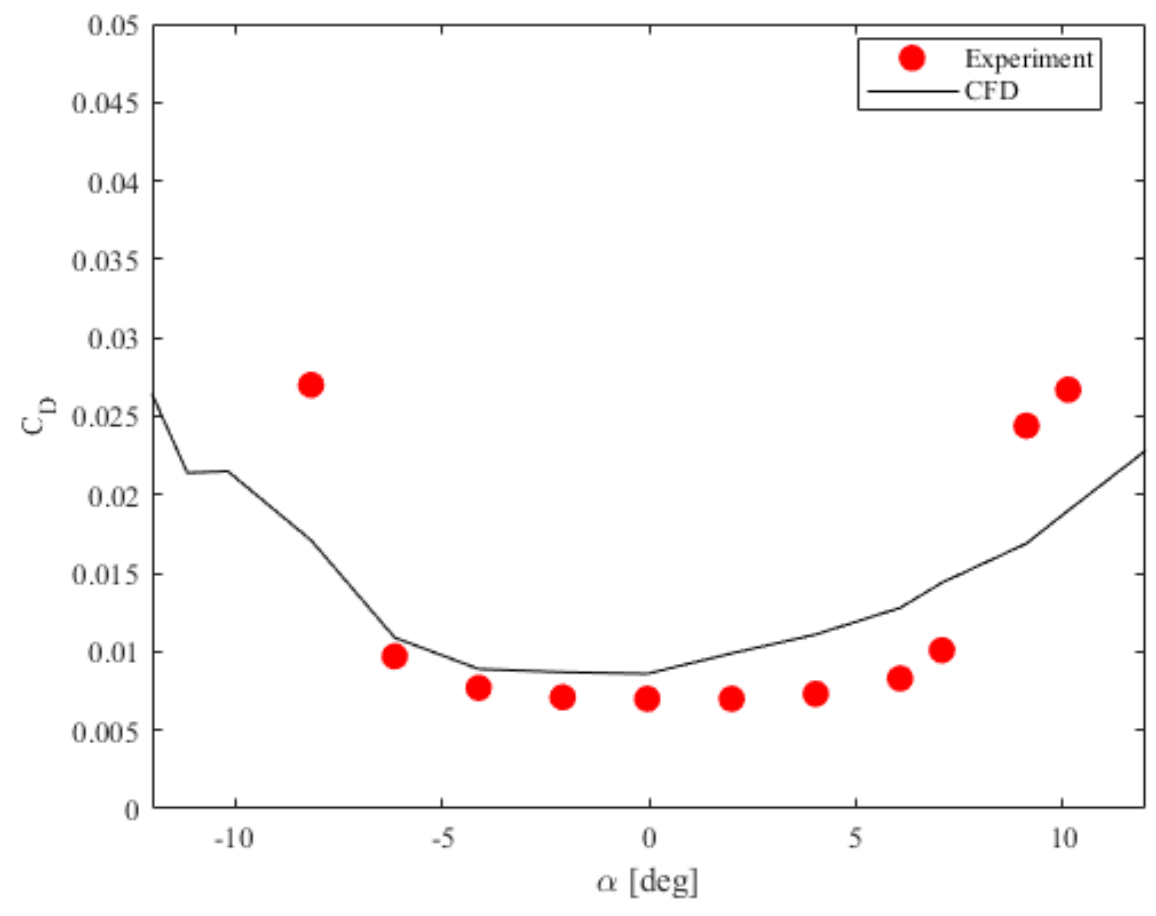

Fig. 4. Drag coefficient vs. angle of attack.

Lift and drag aerodynamic coefficients are computed based on the pressure coefficient distributions. The dimensionless pressure coefficient $C_{P}$ is defined as:

$$
C_{P}=\frac{p-p_{r e f}}{q_{r e f}}
$$

where: $p$ is the static pressure; $p_{r e f}$ is the reference pressure and $q_{r e f}$ is the reference dynamic pressure given as:

$$
q_{\text {ref }}=\frac{1}{2} \rho V^{2}
$$

Figure 5 presents the static pressure distributions obtained using the SST Transition turbulence model. The results are given for three angles of attack: $-6.14^{\circ} ;-0.04^{\circ}$ and $7.08^{\circ}$. The numerical results are compared with the experimental data. As it can be observed from these figures, the level of agreement between numerical and experimental results is very high. 

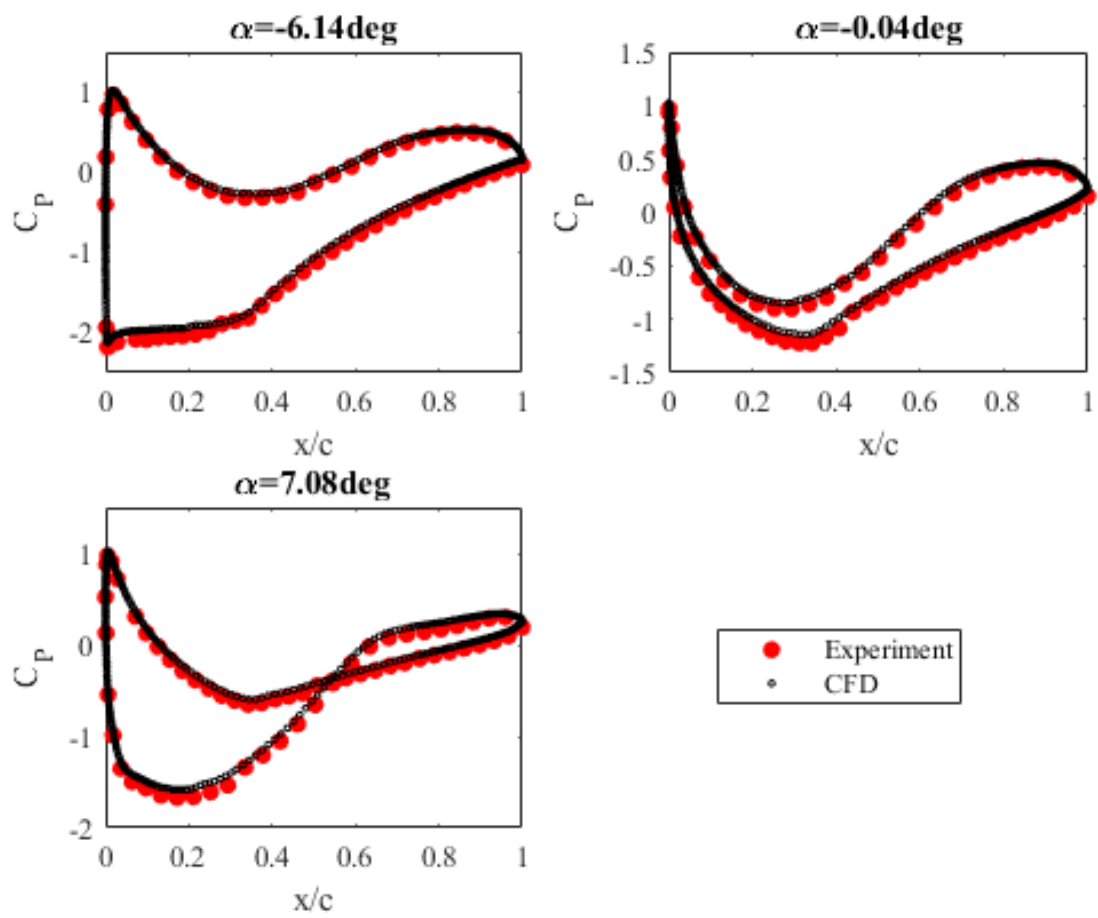

Fig. 5. Static pressure coefficient at three angles of attack.

The static pressure distributions are presented in Fig. 6 using the contour maps. The results are given for three angles of attack: $6.14^{\circ} ;-0.04^{\circ}$ and $7.08^{\circ}$. The range of the static pressure is the same for the all angles of attack. The blue colour on the figures corresponds to the pressure below the reference pressure whereas the red and yellow colours indicate the high pressures. Figure 7 shows the contours of the velocity magnitude around the DU-91-W2-250 airfoil for the same three angles of attack. The range of the velocity is the same for all investigated cases. This figure presents the position of the impact point depending on the angle of attack. For incompressible flows the Mach number is less than 0.3. Figure presents the Mach number distributions around the airfoil for the three angles of attack. At all presented contour maps, the Mach number of 0.3 has been exceeded locally.

a)

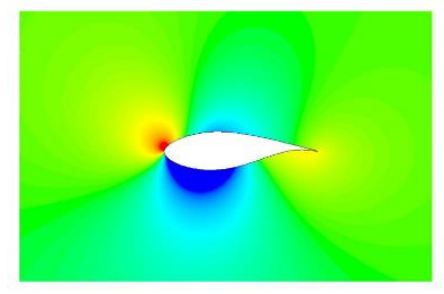

$-5000$ b)

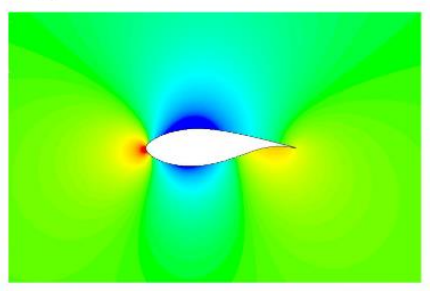

$-1000$ c)

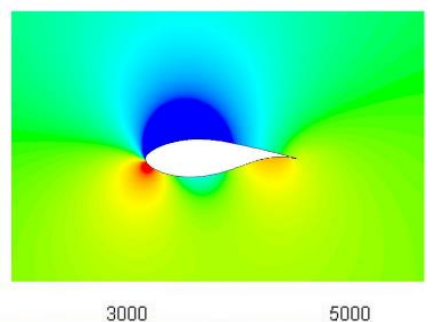

Fig. 6. Contours of static pressure at three angles of attack. 
a)

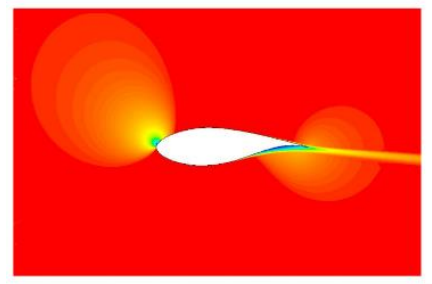

0 b)

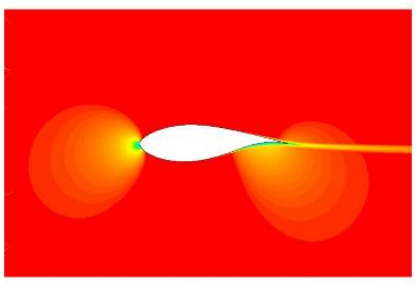

40

60 c)

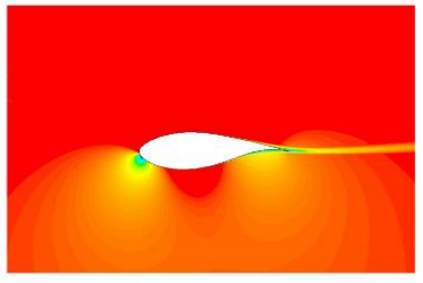

80

100 ?

Fig. 7. Contours of velocity magnitude at three angles of attack.

a)

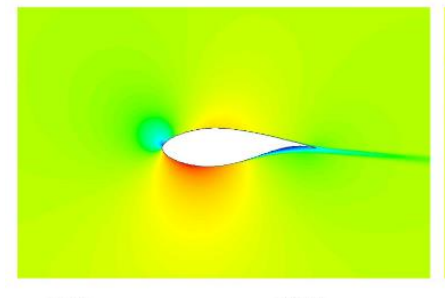

0.00 b)

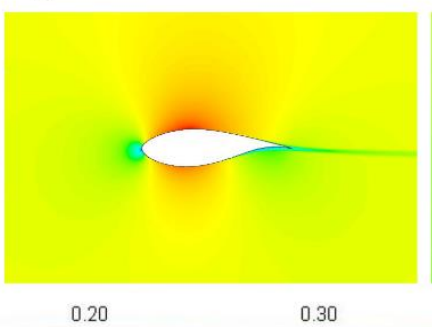

c)

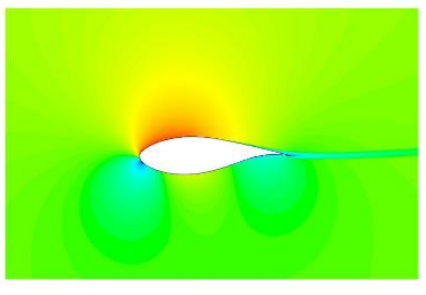

0.40 0.49

Fig. 8. Contours of Mach number at three angles of attack.

\section{Conclusions}

The purpose of the paper was the analysis of the flow past the DU-91-W2-250 under high Reynolds number airfoil using RANS solver. The presented investigations are a part of more extensive numerical and experimental research on a new type of vortex generators for horizontal-axis wind turbine blades. Studies have shown that the SST Transition turbulence models gives satisfactory results of the lift and drag coefficients as well as the static pressure distributions for the angle of attack in the range between the critical angles.

The presented numerical computations were performed in the Interdisciplinary Centre for Mathematical and Computational Modelling of the Warsaw University, Grant No. GA65-29.

\section{References}

1. J.L. Tangler, D.M. Somers, NREL airfoil families for HAWT's, WINDPOWER'95, Washington D.C. Proc., 117-123 (1995)

2. W.J. Zhu, W.Z. Shen, J.N. Sørensen, Integrated airfoil and blade design method for large wind turbines, Renewable Energy, 70, 172-183 (2014)

3. W.A. Timmer, R.P.J.O.M. van Rooij, Summary of the Delft University Wind Turbine Dedicated Airfoils, Journal of Solar Energy Engineering, 125, 488-496 (2003)

4. M.O.L. Hansen, C.M. Velte, S. Øye, R. Hansen, N.N. Sørensen, J. Madsen, R. Mikkelsen, Aerodynamically shaped vortex generators, Wind Energy, 19, 563-567 (2016) 
5. P. Fuglsang, C. Bak, Status of the Risø wind turbine airfoils, presented at the European Wind Energy Conference, Madrid (2003)

6. F. Bertagnolio, N. Sørensen, J. Johansen, P. Fuglsang, Wind Turbine Airfoil Catalogue, Forskningscenter Risoe. Risoe-R; No. 1280(EN) (2001)

7. H. Sarlak Chivaee, Large Eddy Simulation of an SD7003 Airfoil: Effects of Reynolds number and Subgrid-scale modeling, Journal of Physics: Conference Series, 854, (2017)

8. P. Lichota, M. Lasek: Maximum Likelihood Estimation: A method for flight dynamics - Angle of attack estimation, 14th International Carpathian Control Conference, Rytro, 218-221, (2013) 